\title{
Review Article \\ Roles of Protein Synthesis Elongation Factor EF-Tu in Heat Tolerance in Plants
}

\author{
Jianming Fu, ${ }^{1}$ Ivana Momčilović, ${ }^{2}$ and P. V. Vara Prasad ${ }^{3}$ \\ ${ }^{1}$ USDA-ARS-Hard Winter Wheat Genetics Research Unit, 4008 Throckmorton Hall, Kansas State University, Manhattan, \\ KS 66506, USA \\ ${ }^{2}$ Department of Plant Physiology, Institute for Biological Research "Siniša Stanković”, University of Belgrade, \\ Bul. despota Stefana 142, Belgrade 11060, Serbia \\ ${ }^{3}$ Department of Agronomy, Kansas State University, Manhattan, KS 66506, USA
}

Correspondence should be addressed to Jianming Fu, jianming.fu@ars.usda.gov

Received 6 January 2012; Accepted 23 March 2012

Academic Editor: Scott Heckathorn

Copyright (C) 2012 Jianming Fu et al. This is an open access article distributed under the Creative Commons Attribution License, which permits unrestricted use, distribution, and reproduction in any medium, provided the original work is properly cited.

\begin{abstract}
EF-Tu proteins of plastids, mitochondria, and the cytosolic counterpart EF- $1 \alpha$ in plants, as well as EF-Tu proteins of bacteria, are highly conserved and multifunctional. The functions of EF-Tu include transporting the aminoacyl-tRNA complex to the A site of the ribosome during protein biosynthesis; chaperone activity in protecting other proteins from aggregation caused by environmental stresses, facilitating renaturation of proteins when conditions return to normal; displaying a protein disulfide isomerase activity; participating in the degradation of $\mathrm{N}$-terminally blocked proteins by the proteasome; eliciting innate immunity and triggering resistance to pathogenic bacteria in plants; participating in transcription when an E. coli host is infected with phages. EF-Tu genes are upregulated by abiotic stresses in plants, and EF-Tu plays important role in stress responses. Expression of a plant EF-Tu gene confers heat tolerance in E. coli, maize knock-out EF-Tu null mutants are heat susceptible, and over-expression of an EF-Tu gene improves heat tolerance in crop plants. This review paper summarizes the current knowledge of EF-Tu proteins in stress responses in plants and progress on application of EF-Tu for developing crop varieties tolerant to abiotic stresses, such as high temperatures.
\end{abstract}

\section{Introduction}

Protein synthesis elongation factor $\mathrm{Tu}(\mathrm{EF}-\mathrm{Tu})$ is a protein that plays a central role in the elongation phase of protein synthesis in bacteria and organelles including mitochondria and plastids in plants (Figure 1). The cytosolic homolog of EF-Tu in plants is EF- $1 \alpha$. The polypeptide elongation cycle proceeds in three steps: (1) EF-Tu binds GTP and aminoacyltRNA, which leads to the codon-dependent placement of this aminoacyl-tRNA at the A site of the ribosome, GTP hydrolysis, and release of EF-Tu-GDP from the ribosome; (2) EF-Ts (elongation factor Ts) facilitates the exchange of EFTu-bound GDP for GTP; (3) upon the peptide bond formation, EF-G (elongation factor G) translocates the mRNA one codon to allow for the arrival of the new aminoacyltRNA in the A site [1]. EF-Tu and EF-Ts were first isolated as components of so-called factor $\mathrm{T}$ (transfer), and labeled as thermounstable (Tu) and thermostable (Ts) fractions [2], respectively. This comparison of thermostability is questionable because later on EF-Tu has been proved to endure hightemperature treatments $[3,4]$, especially when complexed with nucleotide factors, GTP or GDP [5].

EF-Tu belongs to the group of the translational GTPases or G-Proteins, which also include EF-G and the initiation factor IF2 [6]. All GTPases require hydrolysis of the source of energy, GTP, for large conformational changes related to their functions, switching from relatively closed to open conformations [7]. EF-Tu-GTP has relatively closed conformation, while EF-Tu-GDP has an open conformation.

Plastid EF-Tu is a member of a highly conserved, multigene family, and shows a higher sequence similarity to prokaryotic EF-Tu than to eukaryotic EF-1 $\alpha$, reflecting the EF-Tu origin via ancient endosymbiotic events between cyanobacteria and eukaryotic hosts [8]. E. coli EF-Tu shows $67 \%$ identity and $80 \%$ similarity to plastid EF-Tu in rice and Arabidopsis (Figure 2). Plastid EF-Tu is encoded by the 


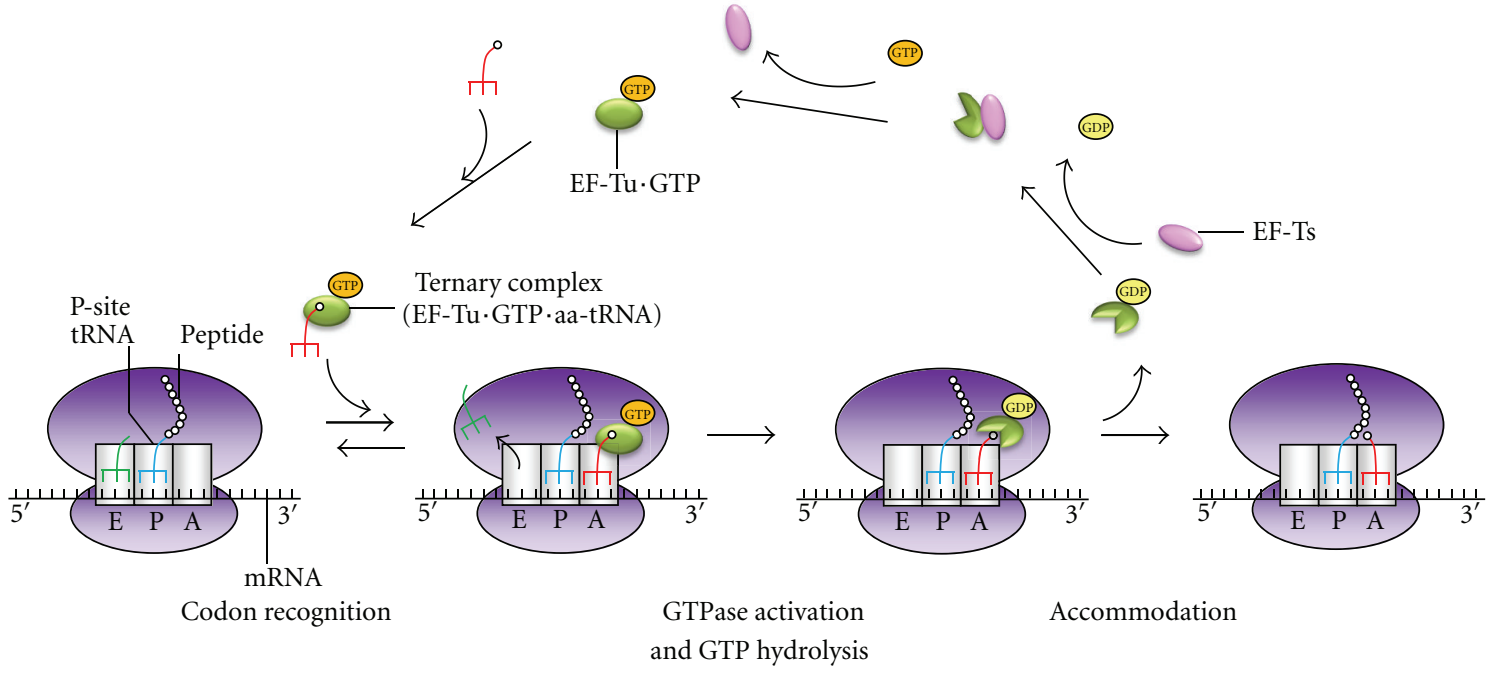

FIgURE 1: The role of EF-Tu in the elongation phase of protein synthesis. EF-Tu-GTP (binary complex) binds aminoacyl-tRNA to form a ternary complex of EF-Tu.GTP-aa-tRNA, which leads to the codon-dependent placement of this aminoacyl-tRNA at the A site of the ribosome. Correct codon-anticodon pairing activates the GTPase centre of the ribosome, which causes GTP hydrolysis, change in EF-Tu conformation and release of the EF-Tu.GDP from aminoacyl end of the tRNA. EF-Ts (elongation factor Ts) facilitates the exchange of EF-Tu-bound GDP for GTP.

plastid genome in most photosynthetic lower eukaryotes, including Euglena gracilis [9], Chlamydomonas reinhardtii [10], and Chlorella vulgaris [11]. In higher plants, this 45$46 \mathrm{kDa}$ plastid protein is encoded by the nuclear genome, synthesized in the cytosol, and then targeted to the plastid. A single plastid EF-Tu gene (tufA) is identified in Arabidopsis thaliana [10], Oryza sativa [12], and geranium [13], whereas multiple copies of the EF-Tu gene exist in the nuclear genome of other plants, ranging from two genes ( $t u f A$ and $t u f B$ ) in Nicotiana sylvestris [14] to four genes (tufA1, tufA2, tufB1, and tufB2) in Glycine max [15].

In addition to the functions in the protein biosynthesis, EF-Tu also plays important roles in responses to both abiotic and biotic stresses in plants. This paper reviews the current knowledge of EF-Tu and EF- $1 \alpha$ with respect to both abiotic and biotic stresses in plants, and the progress in modulating expression of EF-Tu for developing crop varieties with stress tolerance.

\section{EF-Tu Gene Expression Is Regulated by Abiotic Stresses in Plants}

EF-Tu expression has been studied in several plant species in response to different environmental stresses including high and low temperatures, salinity, water deficit, and pollutants. The heat-induced expression and accumulation of plastid EF-Tu, initially reported as $45-\mathrm{kD}$ HSP (heat shock protein), was first reported in experiments with heat tolerant maize line ZPBL1304 [16]. Further investigation identified three heat inducible EF-Tu isoforms/polypeptides (pI 5.1-5.6) in this maize line [17]. The positive correlation between plastid EF-Tu accumulation and heat-tolerant phenotype was reported for several maize hybrids [18]. Plastid EF-Tu synthesis and accumulation has been induced both under heat stress and a combination of heat and drought stress [16, $19,20]$. A recent study in winter wheat has shown that EF-Tu gene expression is upregulated under heat stress conditions, and wheat cultivars that accumulate more EF-Tu protein display better tolerance to heat stress [21]. Heat stressinduced expression/accumulation of both EF-Tu and EF- $1 \alpha$ has been observed in mature plants of spring wheat cultivars [22]. In pea, the expression of the plastid EF-Tu gene tufA is upregulated by light and by low temperatures [23]. Chung et al. [24] used subtractive suppression hybridization to isolate the soybean EF- $1 \alpha$ gene, SLTI100, which is induced by low temperature, high salt, or drought stress. The authors proposed that soybean EF- $1 \alpha$ may play an important role in translation regulation during abiotic stresses. There are also several reports about cold stress-related expression of EF- $1 \alpha$ in barley and maize $[25,26]$.

There is growing evidence about abiotic stress-related EF-Tu expression obtained by a proteomics approach. Proteomic analyses of cold and heat stress responses in rice have identified plastid EF-Tu as an upregulated protein $[27,28]$. Proteomic characterization of Phragmites communis ecotypes has shown major differences for the proteins included in protein biosyntheses [29] and significantly higher EF-Tu accumulation in desert dune compared to swamp ecotype. EF-Tu is also induced by compound 1,2,4-trichlorobenzene, an important component of organochlorine pesticides and pollutant which can be accumulated in plants [30].

\section{EF-Tu Proteins Display Chaperone Activities}

When soluble proteins are in their native conformations, the hydrophobic residues are buried inside. Environmental stresses, such as high temperatures and chemicals, such as acids, can change protein conformations, causing 


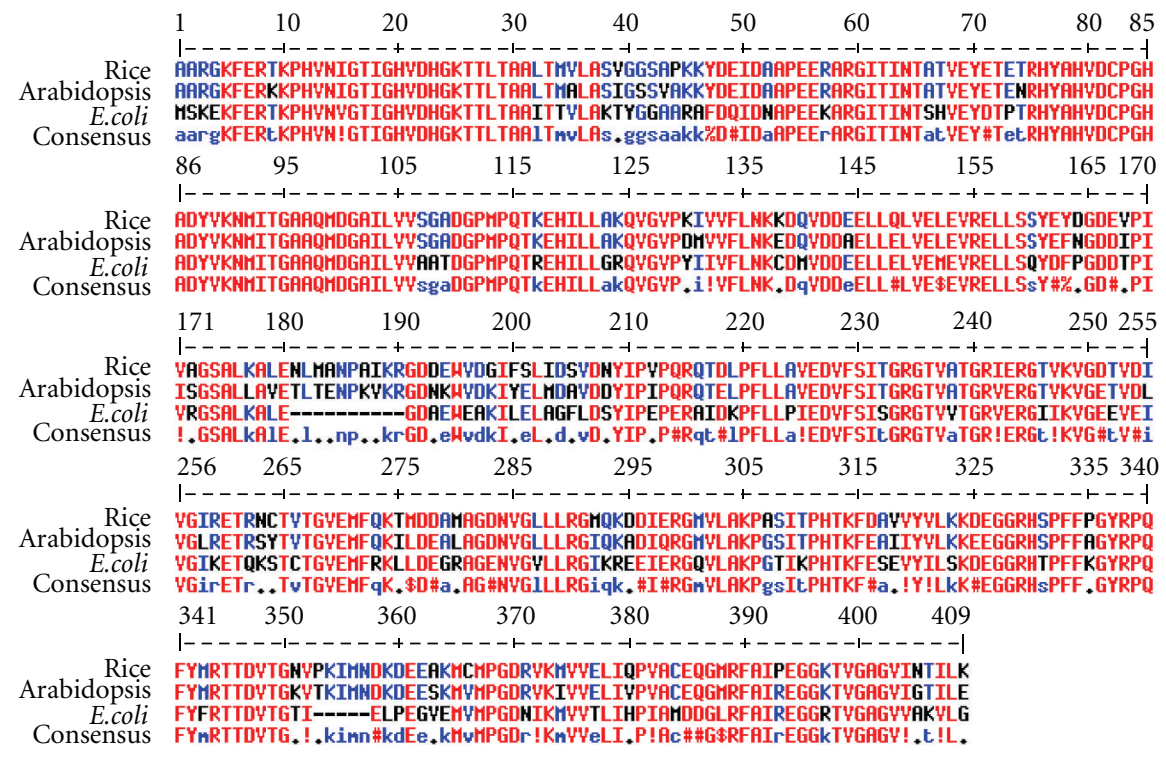

FIgUre 2: The alignment of EF-Tu amino acid sequences. The alignment was conducted based on MULTALIN (http://multalin .toulouse.inra.fr/multalin/multalin.html). The chloroplast targeting sequence was predicted based on Emanuelsson et al. ([31], http://www.cbs.dtu.dk/services/ChloroP/) and then removed before the alignment. Rice (GenBank accession no. AAF15312), Arabidopsis (NP193769), and E. coli (AAA50993). The identities and similarities were calculated based on BLASTP (http://blast.ncbi.nlm.nih.gov/ Blast.cgi?PROGRAM=blastp\&BLAST_PROGRAMS=blastp\&PAGE_TYPE=BlastSearch\&SHOW_DEFAULTS=on\&BLAST_SPEC=blast2seq\& LINK_LOC=blasttab\&LAST_PAGE=blastn\&BLAST_INIT=blast2seq).

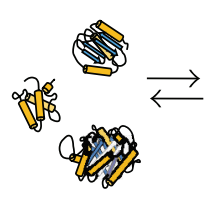

Native form

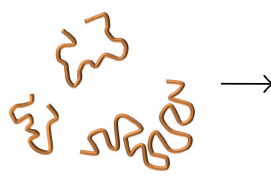

Unfolded form

(a)

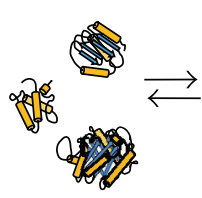

Native form

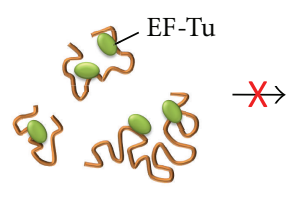

Unfolded form

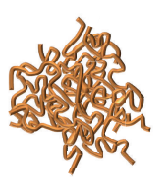

Aggregates

(b)

FIGURE 3: Proposed chaperone-like activity of EF-Tu in plant abiotic stress response. Abiotic stresses can affect protein native conformation and cause protein unfolding (denaturation). Unfolded forms are characterized by exposed hydrophobic residues which promote protein hydrophobic interactions and formation of protein aggregates. Protein denaturation due to stress is reversible unless followed by aggregation. (a) Denatured proteins form aggregates without chaperones, for example, EF-Tu, present. (b) EF-Tu binds to the exposed hydrophobic residues, prevents protein aggregation, and facilitates proteins to return to their native conformation when conditions return normal. In a manner similar to small HSPs, EF-Tu does not require presence of other chaperones or GTP (ATP) for chaperone activity.

exposure of the hydrophobic residues (denaturation). Protein denaturation due to heat shock is reversible unless followed by aggregation [32]. Interactions of exposed residues among denatured protein molecules cause precipitation (aggregation), an irreversible process (Figure 3(a)). Protein chaperones bind to the exposed hydrophobic residues, preventing aggregation, and facilitating the proteins to return to their native conformation when conditions return normal (Figure 3(b)). The chaperone activity of elongation factors was first reported for bacterial EF-Tu. Kudlicki et al. [33] observed that EF-Tu from Thermus thermophilus promotes renaturation of chemically denatured rhodanese, and that the addition of GTP and EF-Ts increased the refolding of denatured proteins. A striking feature of EF-Tu is the large conformational difference between the form of the protein when it binds to GDP or GTP. EF-Tu-GTP has relatively closed conformation, while EF-Tu-GDP has an open conformation. According to Kudlicki et al. [33], flexing of the EF-Tu conformation may be a critical component for refolding of denatured proteins. Another illustration of EFTu chaperone activity is the ability of the E. coli elongation factor in refolding urea-denatured citrate synthase and $\alpha$ glucosidase, and to form stable complexes with unfolded proteins. Bacterial EF-Tu has been found to protect citrate synthase from thermal aggregation in a concentrationdependent manner [5]. The authors reported that the GDPbound form of EF-Tu is much more active than EF-Tu-GTP in stimulating protein renaturation, which is somewhat in dispute with findings of Kudlicki et al. [33]. Recent findings on mammalian mitochondrial EF-Tu, however, suggest that 
EF-Tu promotes refolding of an acid-denaturated green fluorescent protein in a GDP/GTP-independent manner [34]. The authors have also analyzed bacterial and mitochondrial EF-Tu activity according to the methods of Kudlicki et al. [33] and Caldas et al. [5] and concluded that both EF-TuGDP and EF-Tu-GTP are able to interact with unfolded proteins, EF-Tu-GTP may interact with unfolded proteins to a somewhat lesser extent than EF-Tu-GDP, and flexing between EF-Tu-GTP (closed) and EF-Tu-GDP (open) conformation is not required for the chaperone activity of EF-Tu. Recently, chaperone properties of mitochondrial EF-Tu and A. thaliana EF- $1 \alpha$ (AtEF-1 $\alpha$ ) were described in preventing citrate synthase and malate dehydrogenase from thermal aggregation, respectively [34, 35]. Suzuki et al. [34] have also investigated the interaction between the mitochondrial EF$\mathrm{Tu}$ and the newly synthesized peptides from mitochondrial ribosomes under heat stress conditions, which has led to a hypothesis about the possible role of EF-Tu chaperone activity in the quality control of misfolded newly synthesized polypeptides in mitochondria. The role of plastid EF-Tu in preventing protein aggregation is first reported in a study on maize plastid EF-Tu [4] (Figure 3(b)). Precursor of maize plastid EF-Tu (pre-EF-Tu) protects citrate synthase and malate dehydrogenase from thermal aggregation and inactivation without requirements for the presence of other chaperones or GTP (ATP), in a manner similar to some small HSPs [36]. During heat stress, small HSPs interact with substrate proteins in an ATP-independent manner, preventing the substrates from aggregation, but the subsequent refolding during recovery is usually facilitated by other ATPdependent chaperones. Chaperone activity of EF-Tu is also confirmed by chaperone assays in which native wheat preEF-Tu, as well as recombinant and native maize pre-EF$\mathrm{Tu}$, were shown to reduce thermal aggregation of plastid protein Rubisco activase [37]. A previous study had shown that plastid stromal proteins from maize with higher levels of EF-Tu display lower thermal aggregation than plastid stromal proteins from maize with lower levels of EF-Tu [38]. These findings support the hypothesis that maize EF-Tu plays a role in heat tolerance by acting as a molecular chaperone and protecting chloroplast stromal proteins from thermal damages.

In addition to the ability to prevent aggregations of denatured proteins caused by environmental stresses and chemicals, the chaperone-like activities of EF-Tu also include other actions. EF-Tu is an essential host-donated subunit of the replicative complex of $\mathrm{Q} \beta$ phage and interacts with the transcriptional apparatus participating in the synthesis of RNA $[39,40]$. A recent study has shown that host EF$\mathrm{Tu}$ and EF-Ts are involved in the maintenance of the active $\mathrm{Q} \beta$ replicase complex and that EF-Tu : Ts in the $\mathrm{Q} \beta$ replicase may modulate the RNA folding and structure [41]. EF-Tu displays protein disulfide isomerase activity in vitro, catalyzing disulfide bond formation, isomerization, and reduction of proteins [42]. Both EF-Tu and EF- $1 \alpha$ participate in the degradation of ubiquitin N-terminally blocked proteins [43]. EF- $1 \alpha$ interacts with the actin filaments and microtubules both in vitro and in vivo, regulating cytoskeletal organization and cell morphology [44-48].
The protein/peptide-binding motifs of EF-Tu are examined with several protein substrates using cellulose-bound peptide scans or interactions in solution [49]. EF-Tu is found in interacting preferentially with hydrophobic regions of substrate proteins, a strategy used by classic chaperones in preventing aggregation of their substrate proteins [50]. The study of Hotokezaka et al. [51] has shown that part of the EF$1 \alpha$ distal to peptidyltransferase center interacts with nascent and unfolded polypeptides, and that EF- $1 \alpha$ does not interact with correctly folded proteins. Site-directed mutagenesis in EF- $1 \alpha$ NKXD motif, important for binding of GTP and for the conventional role in protein synthesis, does not suppress the chaperone activity of the EF- $1 \alpha$ protein [35]. Also, deletion experiments have shown that the central domain of EF- $1 \alpha$ is essential for the chaperone activity. All these findings suggest that EF-Tu and EF- $1 \alpha$, for performing their chaperone function, use different sites than ones involved in the conventional role in the translation.

\section{Expression of a Plant EF-Tu Gene Enhances Heat Tolerance in E. coli}

The effect of EF-Tu overexpression on development of organismal heat tolerance is first examined in E. coli [52]. A gene for maize plastid EF-Tu (Zmeftu1) is isolated from a cDNA library constructed using mRNA from aerial tissues of maize line B73 exposed first to drought stress and then to $45^{\circ} \mathrm{C}$-heat stress [17]. The pTrcHis2A vector carrying Zmeftul is used to transform competent $E$. coli cells of the strain $\mathrm{DH} 5 \alpha$. E. coli transformed with a maize EF-Tu expression construct (pTrcHis2A-Zmeftu1) is exposed to a high temperature of $55^{\circ} \mathrm{C}$ and viability assessed at $37^{\circ} \mathrm{C}$. Analysis of the E. coli protein extracts has shown that the maize EF-Tu protein is produced at a high level, and the EF-Tu proteins is in the soluble form in the bacterial cells. Significantly, much more E. coli cells induced to produce the recombinant EF-Tu survive high-temperature exposure than their noninduced counterparts and nontransformed control cells, demonstrating that the maize EF-Tu is involved in the development of heat tolerance.

\section{Knock-Out EF-Tu Null Mutants Are Heat Susceptible in Crop Plants}

A search on a maize genome database identifies two plastid EF-Tu DNA sequences (http://www.ncbi.nlm.nih.gov/sites/ entrez? $\mathrm{db}=$ genomeprj\&cmd=Retrieve\&dopt=Overview\&list _uids=9514). Genomic DNA blotting also shows two genes (copies) of plastid EF-Tu in maize genome (Fu et al., in preparation). A screening of transposon-mutagenized maize library TUSC (Trait Utility System for Corn) has identified and led to the isolation of a maize plastid EF-Tu null mutant in which a $5 \mathrm{~kb}$ transposon $\mathrm{Mu} 9$ is inserted in one of the two plastid EF-Tu gene sequences. The mutant has reduced EF-Tu protein level and reduced heat tolerance [53]. We have crossed the homozygous mutant with its wild type and investigated possible cosegregation between the heat-induced accumulation of EF-Tu and the heat tolerant 
phenotype in $F_{2}$ progeny. Heat tolerance and endogenous levels of EF-Tu are investigated. Heat tolerance is assessed by examining damages to thylakoid membranes and measuring the shoot dry mass after the recovery from the heat stress. A significant positive correlation has been observed between the endogenous levels of EF-Tu and the plant tolerance to heat stress; $F_{2}$ plants with higher levels of EF-Tu display better recovery, providing genetic evidence linking plastid EF-Tu and the maize heat tolerant phenotype (Fu et al., in preparation).

Maize plastid EF-Tu promoters are enriched in abiotic stress response elements (REs), including heat, low temperature, drought, and salinity REs (Fu et al., in preparation). This may explain our findings that the maize plastid EF-Tu protein is upregulated by heat and by the combination of heat and dehydration [16]. The possibilities if the eftu genes are up-regulated by salinity and low temperature have not been tested in maize. The existence of low temperature REs in the promoter is somewhat unexpected due to the fact that EF-Tu protein is accumulated by high temperatures. It would be interesting to test if maize eftu gene expression is upregulated under low temperature conditions. The presence of diverse abiotic elements in the promoters of the eftu indicates the involvement of this gene in diverse abiotic responses. In fact, any abiotic stress, if sufficiently intense, will result in nonnative conformations (denaturation) of proteins [54]. Thus, molecular chaperones, such as plastid EF-Tu, may be required in facilitating the recovery of denatured proteins.

\section{Overexpression of an EF-Tu Gene Improves Heat Tolerance in Crop Plants}

EF-Tu plays an important role in heat tolerance. Fu et al. [55] have hypothesized that overexpression of an EF-Tu gene may enhance heat tolerance in crop plants and tested this hypothesis by introducing a maize plastid EF-Tu gene (Zmeftu1) into two cultivars of hexaploid wheat: Bobwhite (BW) and Xinchun 9 (XC9). Twenty four transgenic cell lines have been regenerated and grown in the greenhouse, and 23 lines have produced T1 seeds. Molecular analyses (PCR and genomic DNA blotting) of transgenic plants have confirmed the stable and inheritable insertion of the transgene in the wheat genome. The transgenic cell lines are independent events. Also, wheat genome appears to harbor 3 plastid EF-Tu genes (copies) as genomic DNA blots of two nontransgenic controls (BW and XC9), probed with maize EF-Tu cDNA, have showed three hybridization bands. These hybridization bands also indicate a high similarity between maize and wheat EF-Tu gene sequences. Indeed, an alignment of a wheat plastid EF-Tu cDNA sequence (TC264979) from wheat cDNA (EST) database (http://compbio.dfci.harvard.edu/tgi/) and the maize EFTu cDNA probe sequence shows $88 \%$ identity. At the protein level, these two plastid EF-Tu sequences show $88 \%$ identity and 93\% similarity (BLAST 2 Sequences Program; http://www.ncbi.nlm.nih.gov). RNA blotting analysis has demonstrated that maize EF-Tu mRNA is accumulated at a high level in transgenic wheat. One-dimensional immunoblotting has showed that several transgenic events display significant higher levels of EF-Tu proteins than nontrans-control XC9. Two-dimensional immunoblotting has showed that the maize EF-Tu protein in transgenic wheat appears to be posttranslationally modified because several maize EF-Tu protein spots with different $\mathrm{pI}$ are detected. The overexpression of maize EF-Tu in transgenic wheat does not have any noticeable adverse effects on plant growth and development and does not compromise agronomic performance in nonstress conditions. Thermal aggregation assays have showed that transgenic events with increased expression of plastid EF-Tu display reduced thermal aggregation of leaf proteins. The transgenic plants with increased levels of the plastid EF-Tu have displayed reduced injuries to photosynthetic membranes (thylakoids), enhanced rate of $\mathrm{CO}_{2}$ fixation, and fewer visible signs of heat injuries following exposure to heat stress. The reduced injury and enhanced $\mathrm{CO}_{2}$ fixation is probably contributable to the protection of photosynthetic membranes and photosynthesis-related enzymes under the heat stress. Several high-performance transgenic lines have been advanced to the homozygous stage $[55,56]$. The field trials for testing the performance of these transgenic wheat lines in adverse natural environments are currently underway.

\section{EF-Tu Plays an Important Role in Biotic Stress in Plants}

Plants have evolved sensitive perception systems for discriminating invading microbial pathogens. The molecular basis of such discrimination is the recognition of the pathogenassociated molecular patterns (PAMP) by the plant immune systems. When Kunze et al. [57] mixed crude bacterial extracts with Arabidopsis cell cultures, the plant cell immune response was quickly triggered, indicating that the crude bacterial extracts contain PAMP. Purification of the elicitoractive protein from the bacterial extracts has revealed EF$\mathrm{Tu}$ identity. Based on the immune responses of the plant cells to different fragments of EF-Tu the N-terminal part is identified as the elicitor. Further investigation has pinpointed the $\mathrm{N}$-terminal acetylated peptide of the first 18 amino acids as the fully active inducer of defense responses. The innate immunity of the plant cells induces an oxidative burst and ethylene production and results in resistance to subsequent infection of pathogenic microbes $[57,58]$. These results indicate that EF-Tu plays an important role in biotic stress in plants and that the mechanisms of disease resistance and heat tolerance may crosstalk at the molecular level. It would be interesting to explore the possibility to engineering crop plants to resist both disease and heat stress using EF-Tu gene in the future.

\section{Summary and Perspectives}

The world population grows rapidly. On the other hand, urbanization and soil degradation reduce the agricultural land, and increasing abiotic stress, such as high temperatures due to the global warming, and biotic stress constrain crop 


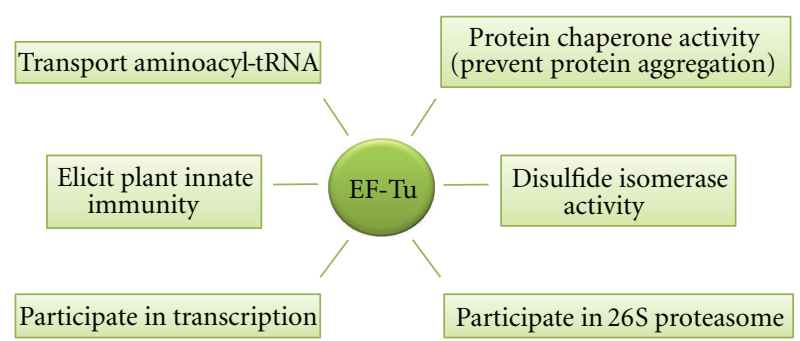

FIgURE 4: EF-Tu proteins are multifunctional. (1) EF-Tu plays a required function during protein synthesis by transporting aminoacyl-tRNA complex and facilitate the complex to bind to the A site of the ribosome; (2) EF-Tu possesses chaperone activity in protecting other proteins from aggregation by binding to the hydrophobic regions of the denatured proteins; (3) EF-Tu displays a protein disulfide isomerase activity; (4) EF-Tu proteins can elicit innate immunity and trigger resistance to pathogenic bacteria; (5) when E. coli is infected with RNA phages, EF-Tu becomes a subunit of the enzyme in the transcriptional apparatus responsible for the RNA replication, suggesting that EF-Tu play a role in transcription; (6) EF-Tu can substitute EF- $1 \alpha$ for participating in the degradation of $\mathrm{N}$ terminally blocked proteins by the $26 \mathrm{~S}$ proteasome.

productivity [59-63]. Thus, identification and application of novel genes for developing crop varieties tolerant (resistant) to abiotic (biotic) stresses might be an efficient way for reducing the pressure of the food demand in the future $[64,65]$.

Plastid EF-Tu, mitochondrial EF-Tu, and the cytosolic counterpart EF- $1 \alpha$ in plants, as well as bacterial EF-Tu, are among the slowest evolving sequences and highly conserved [66]. EF-Tu $(1 \alpha)$ proteins are of multifunctions (Figure 4). One of these functions is to play a central role during protein synthesis by transporting aminoacyl-tRNA complex and facilitating the complex to bind to the A site of the ribosome [1]. Second, EF-Tu possesses chaperone activity in protecting other proteins from aggregation by binding to the hydrophobic regions of the denatured proteins $[4,5]$. Third, EF-Tu displays a protein disulfide isomerase activity. Like other protein disulfide oxidoreductases, such as thioredoxin, protein disulfide isomerase (PDI) and DsbA (Disulfide bond formation, a bacterial version of PDI), EF-Tu catalyzes disulfide bond formation, isomerization, and reduction of proteins (e.g., insulin), depending on the imposed redox potential and the nature of the polypeptide substrates [42]. The isomerase activity requires cysteine residues in the catalysts (enzymes). The cysteine residues in EF-Tu proteins from different sources, including E. coli and plants, are highly conserved, indicating that EF-Tu may play a role in the cysteine-related functions [42]. Fourth, EF-Tu proteins of bacteria including plant pathogenic bacteria, such as Erwinia carotovora, $R$. solanacearum, and A. tumefaciens, can elicit innate immunity and trigger resistance to pathogenic bacteria in plants [57]. Fifth, when E. coli cells are infected with RNA phages, EF$\mathrm{Tu}$ becomes a subunit of the enzyme in the transcriptional apparatus responsible for the RNA replication, suggesting that EF-Tu play a role in transcription [40]. Sixth, EF$1 \alpha$ participates in the degradation of $\mathrm{N}$ terminally blocked proteins by the $26 \mathrm{~S}$ proteasome, a quality control process. EF-Tu can substitute EF- $1 \alpha$ for this function [43].
EF-Tu plays important roles in abiotic stresses, such as heat tolerance. Expression of a maize EF-Tu gene confers heat tolerance in E. coli. Knock-out EF-Tu mutants in maize show less EF-Tu protein levels and less heat tolerant than the wild type. The overexpression of chloroplast EF-Tu in transgenic wheat has led to improved protection of leaf proteins against thermal aggregation, reduced damages to thylakoid membranes and enhanced photosynthetic capability following exposure to heat stress. These results strongly suggest that heat tolerance of wheat, and possibly other crop plants, can be improved by modulating expression levels of plastid EF-Tu [67]. EF-Tu also plays an important role in inducing innate immunity for the plant cells to resist subsequent infection of pathogenic microbes. Therefore, there is the possibility for application of EF-Tu in developing crop varieties both tolerant to abiotic stress and resistant to disease.

\section{Acknowledgments}

The authors are thankful to Dr. Robert Bowden, USDA-ARSHard Winter Wheat Research Genetics Unit, Manhattan, KS for critical reading of the manuscript and Dr. Pradhan Gautam, Department of Agronomy, Kansas State University for helpful discussion. Dr. I. Momčilović acknowledges support from Ministry of Education and Science, Republic of Serbia, Project Grant no. TR31049. This paper is dedicated to the memory of Dr. Zoran Ristic who discovered significant role of the chloroplast EF-Tu in plant heat tolerance. Kansas Agriculture Experiment Station Contribution no: 12-372-J.

\section{References}

[1] B. Riis, S. I. S. Rattan, B. F. C. Clark, and W. C. Merrick, "Eukaryotic protein elongation factors," Trends in Biochemical Sciences, vol. 15, no. 11, pp. 420-424, 1990.

[2] J. Lucas-Lenard, "Protein biosynthesis," Annual Review of Biochemistry, vol. 40, pp. 409-448, 1971.

[3] K. Arai, M. Kawakita, and Y. Kaziro, "Studies on the polypeptide elongation factors from E. coli. V. Properties of various complexes containing EF Tu and EF Ts," Journal of Biochemistry, vol. 76, no. 2, pp. 293-306, 1974.

[4] D. Rao, I. Momcilovic, S. Kobayashi, E. Callegari, and Z. Ristic, "Chaperone activity of recombinant maize chloroplast protein synthesis elongation factor, EF-Tu," European Journal of Biochemistry, vol. 271, no. 18, pp. 3684-3692, 2004.

[5] T. D. Caldas, A. El Yaagoubi, and G. Richarme, "Chaperone properties of bacterial elongation factor EF-Tu," Journal of Biological Chemistry, vol. 273, no. 19, pp. 11478-11482, 1998.

[6] T. Caldas, S. Laalami, and G. Richarme, "Chaperone properties of bacterial elongation factor EF-G and initiation factor IF2," Journal of Biological Chemistry, vol. 275, no. 2, pp. 855$860,2000$.

[7] I. M. Krab and A. Parmeggiani, "EF-Tu, a GTPase odyssey," Biochimica et Biophysica Acta, vol. 1443, no. 1-2, pp. 1-22, 1998.

[8] M. W. Gray, “The endosymbiont hypothesis revisited," International Review of Cytology, vol. 141, pp. 233-357, 1992.

[9] P. E. Montandon and E. Stutz, "Nucleotide sequence of a Euglena gracilis chloroplast genome region coding for the 
elongation factor Tu: evidence for a spliced mRNA," Nucleic Acids Research, vol. 11, no. 17, pp. 5877-5892, 1983.

[10] S. L. Baldauf and J. D. Palmer, "Evolutionary transfer of the chloroplast tufA gene to the nucleus," Nature, vol. 344, no. 6263, pp. 262-265, 1990.

[11] T. Wakasugi, T. Nagai, M. Kapoor et al., "Complete nucleotide sequence of the chloroplast genome from the green alga Chlorella vulgaris: the existence of genes possibly involved in chloroplast division," Proceedings of the National Academy of Sciences of the United States of America, vol. 94, no. 11, pp. 5967-5972, 1997.

[12] J. H. Lee, J. W. Lee, Y. Y. Chung et al., "Cloning and characterization of the chloroplast elongation factor EF-Tu cDNA of Oryza sativa L," Molecules and Cells, vol. 9, no. 5, pp. 484490, 1999.

[13] C. J. Kang, M. G. Lee, Y. S. Cho et al., "Characterization of geranium (Pelargonium graveolens) chloroplast EF-Tu cDNA," Molecules and Cells, vol. 10, no. 5, pp. 579-583, 2000.

[14] Y. Murayama, T. Matsubayashi, M. Sugita, and M. Sugiura, "Purification of chloroplast elongation factor Tu and cDNA analysis in tobacco: the existence of two chloroplast elongation factor Tu species," Plant Molecular Biology, vol. 22, no. 5, pp. 767-774, 1993.

[15] C. Bonny and E. Stutz, "Soybean (Glycine max L.) nuclear DNA contains four tufA genes coding for the chloroplastspecific translation elongation factor EF-Tu," Chimia, vol. 47, no. 6, pp. 247-249, 1993.

[16] Z. Ristic, D. J. Gifford, and D. D. Cass, "Heat shock proteins in two lines of Zea mays L. that differ in drought and heat resistance," Plant Physiology, vol. 97, no. 4, pp. 1430-1434, 1991.

[17] S. K. Bhadula, T. E. Elthon, J. E. Habben, T. G. Helentjaris, S. Jiao, and Z. Ristic, "Heat-stress induced synthesis of chloroplast protein synthesis elongation factor (EF-Tu) in a heattolerant maize line," Planta, vol. 212, no. 3, pp. 359-366, 2001.

[18] Z. Ristic, G. Williams, G. Yang, B. Martin, and S. Fullerton, "Dehydration, damage to cellular membranes, and heat-shock proteins in maize hybrids from different climates," Journal of Plant Physiology, vol. 149, no. 3-4, pp. 424-432, 1996.

[19] Z. Ristic, G. Yang, and S. K. Bhadula, "Two-dimensional gel analysis of $45 \mathrm{ku}$ heat shock proteins from a drought and heat resistant maize line," Journal of Plant Physiology, vol. 154, no. 2, pp. 264-268, 1999.

[20] I. Momcilovic and Z. Ristic, "Expression of chloroplast protein synthesis elongation factor, EF-Tu, in two lines of maize with contrasting tolerance to heat stress during early stages of plant development," Journal of Plant Physiology, vol. 164, no. 1, pp. 90-99, 2007.

[21] Z. Ristic, U. Bukovnik, I. Momčilović, J. Fu, and P. V. Vara Prasad, "Heat-induced accumulation of chloroplast protein synthesis elongation factor, EF-Tu, in winter wheat," Journal of Plant Physiology, vol. 165, no. 2, pp. 192-202, 2008.

[22] U. Bukovnik, J. Fu, M. Bennett, P. V. V. Prasad, and Z. Ristic, "Heat tolerance and expression of protein synthesis elongation factors, EF-Tu and EF-1 $\alpha$, in spring wheat," Functional Plant Biology, vol. 36, no. 3, pp. 234-241, 2009.

[23] B. N. Singh, R. N. Mishra, P. K. Agarwal et al., "A pea chloroplast translation elongation factor that is regulated by abiotic factors," Biochemical and Biophysical Research Communications, vol. 320, no. 2, pp. 523-530, 2004.

[24] E. Chung, C. W. Cho, H. A. So, B. H. Yun, and J. H. Lee, "Differential expression of soybean SLTI100 gene encoding translation elongation factor 1A by abiotic stresses," Journal of Plant Biotechnology, vol. 36, no. 3, pp. 255-260, 2009.
[25] M. A. Dunn, A. Morris, P. L. Jack, and M. A. Hughes, "A lowtemperature-responsive translation elongation factor 1alpha from barley (Hordeum vulgare L.)," Plant Molecular Biology, vol. 23, no. 1, pp. 221-225, 1993.

[26] T. Berberich, K. Sugawara, M. Harada, and T. Kusano, "Molecular cloning, characterization and expression of an elongation factor 1alpha gene in maize," Plant Molecular Biology, vol. 29, no. 3, pp. 611-615, 1995.

[27] S. Cui, F. Huang, J. Wang, X. Ma, Y. Cheng, and J. Liu, "A proteomic analysis of cold stress responses in rice seedlings," Proteomics, vol. 5, no. 12, pp. 3162-3172, 2005.

[28] D. G. Lee, N. Ahsan, S. H. Lee et al., "A proteomic approach in analyzing heat-responsive proteins in rice leaves," Proteomics, vol. 7, no. 18, pp. 3369-3383, 2007.

[29] S. Cui, J. Hu, B. Yang et al., "Proteomic characterization of Phragmites communis in ecotypes of swamp and desert dune," Proteomics, vol. 9, no. 16, pp. 3950-3967, 2009.

[30] C-L. Ge, D-Z. Wan, Z-G. Wang et al., "Response of rice roots to 1, 2, 4-trichlorobenzene stress," Acta Agronomica Sinica, vol. 33, no. 12, pp. 1991-2000, 2007.

[31] http://www.cbs.dtu.dk/services/ChloroP/.

[32] C. Tanford, "Protein denaturation," in Advances in Protein Chemistry, C. B. Anfinsen, J. T. Edsal, M. L. Anson, and F.M. Richards, Eds., pp. 121-282, Academic Press, New York, NY, USA, 1968.

[33] W. Kudlicki, A. Coffman, G. Kramer, and B. Hardesty, "Renaturation of rhodanese by translational elongation factor (EF) Tu: protein refolding by EF-Tu flexing," Journal of Biological Chemistry, vol. 272, no. 51, pp. 32206-32210, 1997.

[34] H. Suzuki, T. Ueda, H. Taguchi, and N. Takeuchi, "Chaperone properties of mammalian mitochondrial translation elongation factor Tu," Journal of Biological Chemistry, vol. 282, no. 6, pp. 4076-4084, 2007.

[35] D. Shin, S. J. Moon, S. R. Park, B. G. Kim, and M. O. Byun, "Elongation factor $1 \alpha$ from $A$. thaliana functions as molecular chaperone and confers resistance to salt stress in yeast and plants," Plant Science, vol. 177, no. 3, pp. 156-160, 2009.

[36] G. J. Lee, N. Pokala, and E. Vierling, "Structure and in vitro molecular chaperone activity of cytosolic small heat shock proteins from pea," Journal of Biological Chemistry, vol. 270, no. 18, pp. 10432-10438, 1995.

[37] Z. Ristic, I. Momčilović, J. Fu, E. Callegari, and B. P. DeRidder, "Chloroplast protein synthesis elongation factor, EF-Tu, reduces thermal aggregation of rubisco activase," Journal of Plant Physiology, vol. 164, no. 12, pp. 1564-1571, 2007.

[38] I. Momcilovic and Z. Ristic, "Localization and abundance of chloroplast protein synthesis elongation factor (EF-Tu) and heat stability of chloroplast stromal proteins in maize," Plant Science, vol. 166, no. 1, pp. 81-88, 2004.

[39] A. A. Travers, R. I. Kamen, and R. F. Schleif, "Factor necessary for ribosomal RNA synthesis," Nature, vol. 228, no. 5273, pp. 748-751, 1970.

[40] T. Blumenthal, T. A. Landers, and K. Weber, "Bacteriophage Q replicase contains the protein biosynthesis elongation factors EF Tu and EF Ts," Proceedings of the National Academy of Sciences of the United States of America, vol. 69, no. 5, pp. 13131317, 1972.

[41] D. Takeshita and K. Tomita, "Assembly of $\mathrm{Q} \beta$ viral RNA polymerase with host translational elongation factors EF-Tu and-Ts," Proceedings of the National Academy of Sciences of the United States of America, vol. 107, no. 36, pp. 15733-15738, 2010 . 
[42] G. Richarme, "Protein-disulfide isomerase activity of elongation factor EF-Tu," Biochemical and Biophysical Research Communications, vol. 252, no. 1, pp. 156-161, 1998.

[43] H. Gonen, C. E. Smith, N. R. Siegel et al., "Protein synthesis elongation factor EF- $1 \alpha$ is essential for ubiquitin- dependent degradation of certain $\mathrm{N}(\alpha)$-acetylated proteins and may be substituted for by the bacterial elongation factor EF-Tu," Proceedings of the National Academy of Sciences of the United States of America, vol. 91, no. 16, pp. 7648-7652, 1994.

[44] F. Yang, M. Demma, V. Warren, S. Dharmawardhane, and J. Condeelis, "Identification of an actin-binding protein from Dictyostelium as elongation factor 1a," Nature, vol. 347, no. 6292, pp. 494-496, 1990.

[45] K. Kandl, R. Munshi, P. Ortiz, G. Andersen, T. Kinzy, and A. Adams, "Identification of a role for actin in translational fidelity in yeast," Molecular Genetics and Genomics, vol. 268, no. 1, pp. 10-18, 2002.

[46] G. Liu, W. M. Grant, D. Persky, V. M. Latham, R. H. Singer, and J. Condeelis, "Interactions of elongation factor $1 \alpha$ with Factin and $\beta$-actin mRNA: implications for anchoring mRNA in cell protrusions," Molecular Biology of the Cell, vol. 13, no. 2, pp. 579-592, 2002.

[47] J. A. Lopez-Valenzuela, B. C. Gibbon, P. A. Hughes, T. W. Dreher, and B. A. Larkins, "eEF1A isoforms change in abundance and actin-binding activity during maize endosperm development," Plant Physiology, vol. 133, no. 3, pp. 1285-1295, 2003.

[48] S. R. Gross and T. G. Kinzy, "Translation elongation factor $1 \mathrm{~A}$ is essential for regulation of the actin cytoskeleton and cell morphology," Nature Structural and Molecular Biology, vol. 12, no. 9, pp. 772-778, 2005.

[49] A. Malki, T. Caldas, A. Parmeggiani, M. Kohiyama, and G. Richarme, "Specificity of elongation factor EF-Tu for hydrophobic peptides," Biochemical and Biophysical Research Communications, vol. 296, no. 3, pp. 749-754, 2002.

[50] J. P. Hendrick and F. U. Hartl, "Molecular chaperone functions of heat-shock proteins," Annual Review of Biochemistry, vol. 62, pp. 349-384, 1993.

[51] Y. Hotokezaka, U. T. Bben, H. Hotokezaka et al., "Interaction of the eukaryotic elongation factor 1A with newly synthesized polypeptides," Journal of Biological Chemistry, vol. 277, no. 21, pp. 18545-18551, 2002.

[52] T. Moriarty, R. West, G. Small, D. Rao, and Z. Ristic, "Heterologous expression of maize chloroplast protein synthesis elongation factor (EF-Tu) enhances Escherichia coli viability under heat stress," Plant Science, vol. 163, no. 6, pp. 10751082, 2002.

[53] Z. Ristic, K. Wilson, C. Nelsen et al., "A maize mutant with decreased capacity to accumulate chloroplast protein synthesis elongation factor $(\mathrm{EF}-\mathrm{Tu})$ displays reduced tolerance to heat stress," Plant Science, vol. 167, no. 6, pp. 1367-1374, 2004.

[54] M. E. Feder and G. E. Hofmann, "Heat-shock proteins, molecular chaperones, and the stress response: evolutionary and ecological physiology," Annual Review of Physiology, vol. 61, pp. 243-282, 1999.

[55] J. Fu, I. Momčilović, T. E. Clemente, N. Nersesian, H. N. Trick, and Z. Ristic, "Heterologous expression of a plastid EFTu reduces protein thermal aggregation and enhances $\mathrm{CO}_{2}$ fixation in wheat (Triticum aestivum) following heat stress," Plant Molecular Biology, vol. 68, no. 3, pp. 277-288, 2008.

[56] J. Fu and Z. Ristic, "Analysis of transgenic wheat (Triticum aestivum L.) harboring a maize (Zea mays L.) gene for plastid EF-Tu: segregation pattern, expression and effects of the transgene," Plant Molecular Biology, vol. 73, no. 3, pp. 339$347,2010$.

[57] G. Kunze, C. Zipfel, S. Robatzek, K. Niehaus, T. Boller, and G. Felix, "The N terminus of bacterial elongation factor Tu elicits innate immunity in Arabidopsis plants," Plant Cell, vol. 16, no. 12, pp. 3496-3507, 2004.

[58] C. Zipfel, G. Kunze, D. Chinchilla et al., "Perception of the Bacterial PAMP EF-Tu by the receptor EFR restricts agrobacterium-mediated transformation," Cell, vol. 125, no. 4, pp. 749760, 2006

[59] J. Levitt, Responses of Plants to Environmental Stress: Chilling, Freezing and High Temperature Stresses, Academic Press, New York, NY, USA, 1980.

[60] J. S. Boyer, "Plant productivity and environment," Science, vol. 218, no. 4571, pp. 443-448, 1982.

[61] S. Peng, J. Huang, J. E. Sheehy et al., "Rice yields decline with higher night temperature from global warming," Proceedings of the National Academy of Sciences of the United States of America, vol. 101, no. 27, pp. 9971-9975, 2004.

[62] D. S. Battisti and R. L. Naylor, "Historical warnings of future food insecurity with unprecedented seasonal heat," Science, vol. 323, no. 5911, pp. 240-244, 2009.

[63] S. P. Long and D. R. Ort, "More than taking the heat: crops and global change," Current Opinion in Plant Biology, vol. 13, no. 3, pp. 241-248, 2010.

[64] H. J. Bohnert, Q. Gong, P. Li, and S. Ma, "Unraveling abiotic stress tolerance mechanisms-getting genomics going," Current Opinion in Plant Biology, vol. 9, no. 2, pp. 180-188, 2006.

[65] S. Kotak, J. Larkindale, U. Lee, P. von Koskull-Döring, E. Vierling, and K. D. Scharf, "Complexity of the heat stress response in plants," Current Opinion in Plant Biology, vol. 10, no. 3, pp. 310-316, 2007.

[66] E. A. Gaucher, M. M. Miyamoto, and S. A. Benner, "Functionstructure analysis of proteins using covarion-based evolutionary approaches: elongation factors," Proceedings of the National Academy of Sciences of the United States of America, vol. 98, no. 2, pp. 548-552, 2001.

[67] J. Fu, I. Momčilović, and V. Prasad, "Molecular bases and improvement of heat tolerance in crop plants," in Heat Stress: Causes, Prevention and Treatments, S. Josipovic and E. Ludwig, Eds., pp. 185-214, Nova Science, USA, 2012. 

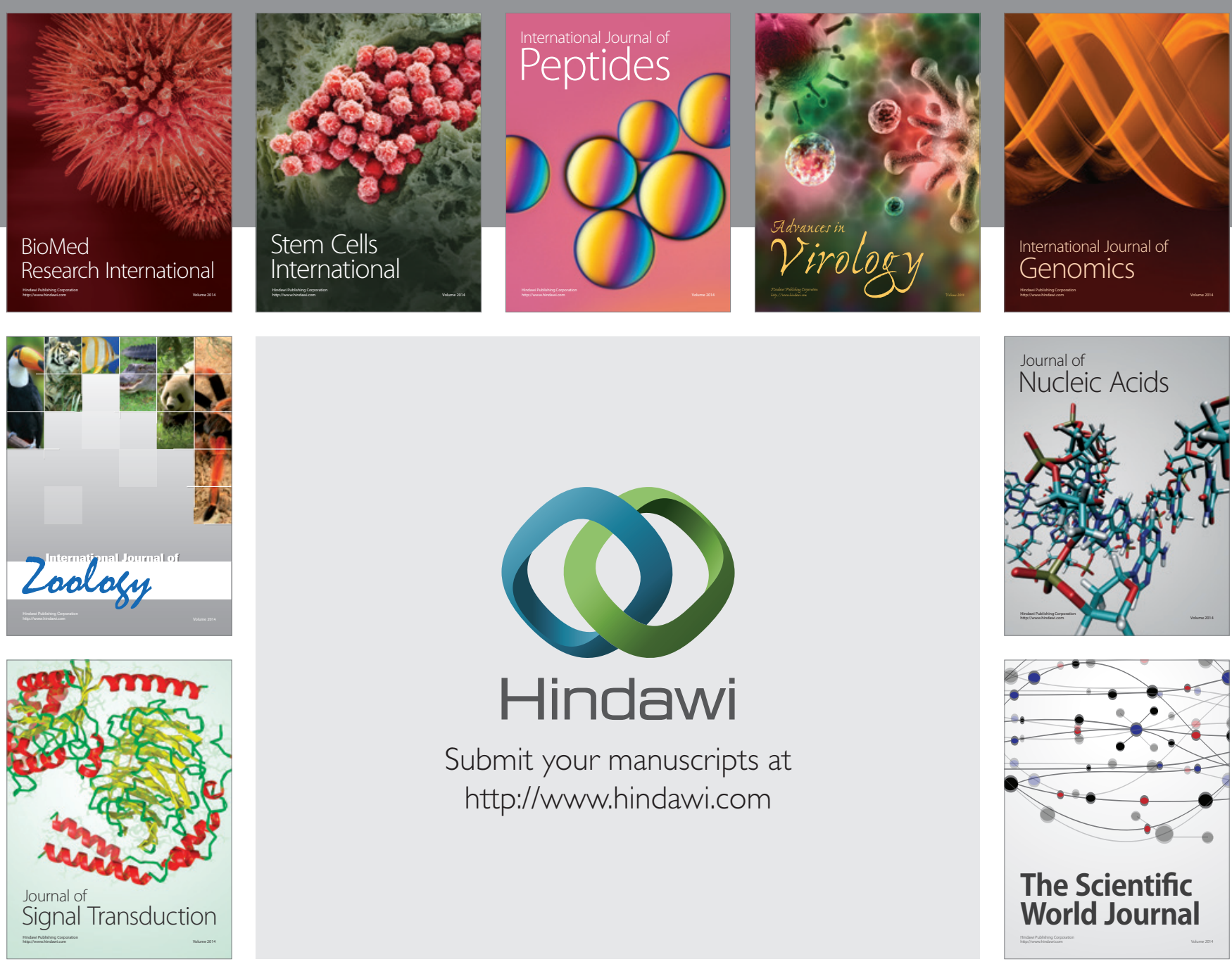

Submit your manuscripts at

http://www.hindawi.com
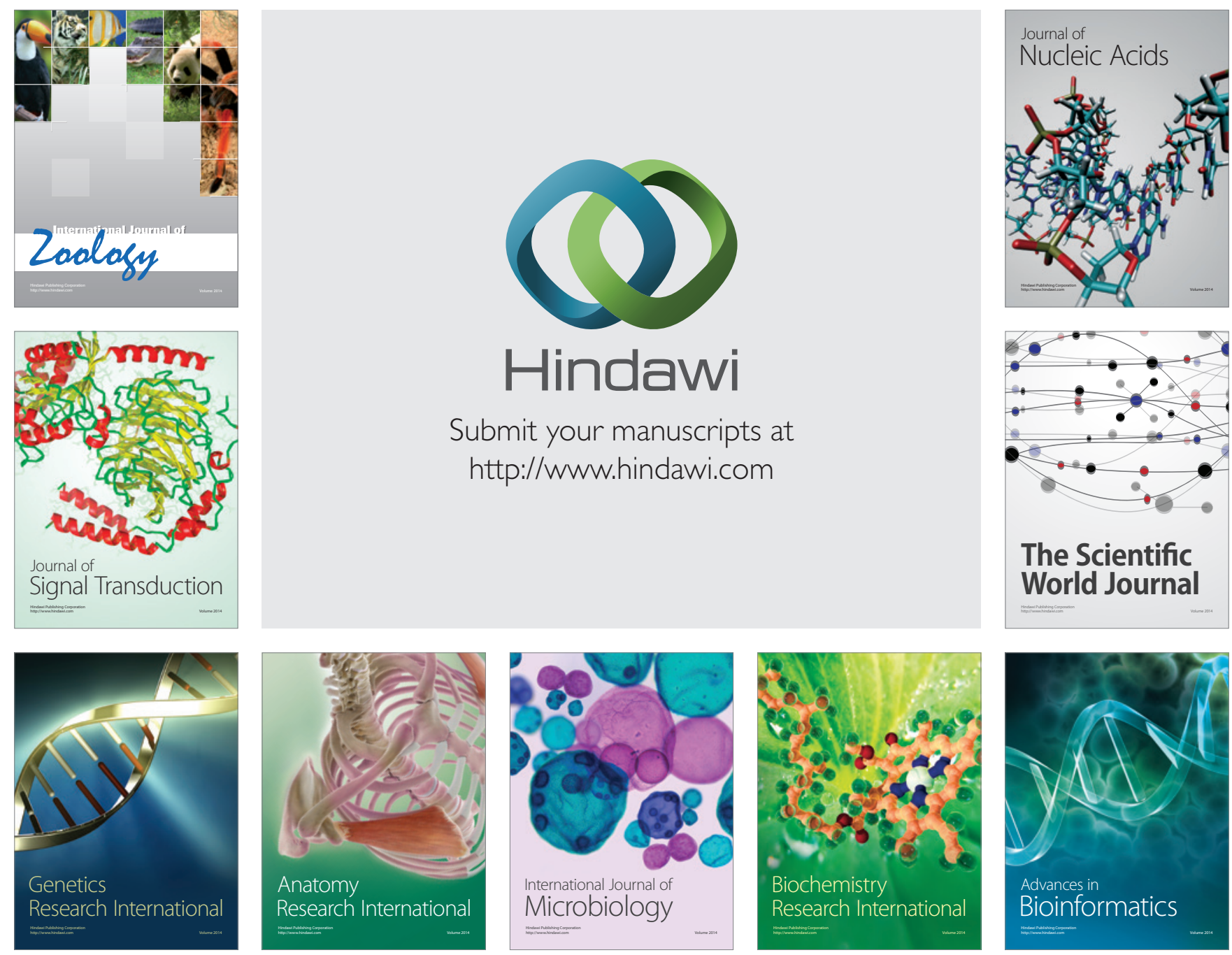

The Scientific World Journal
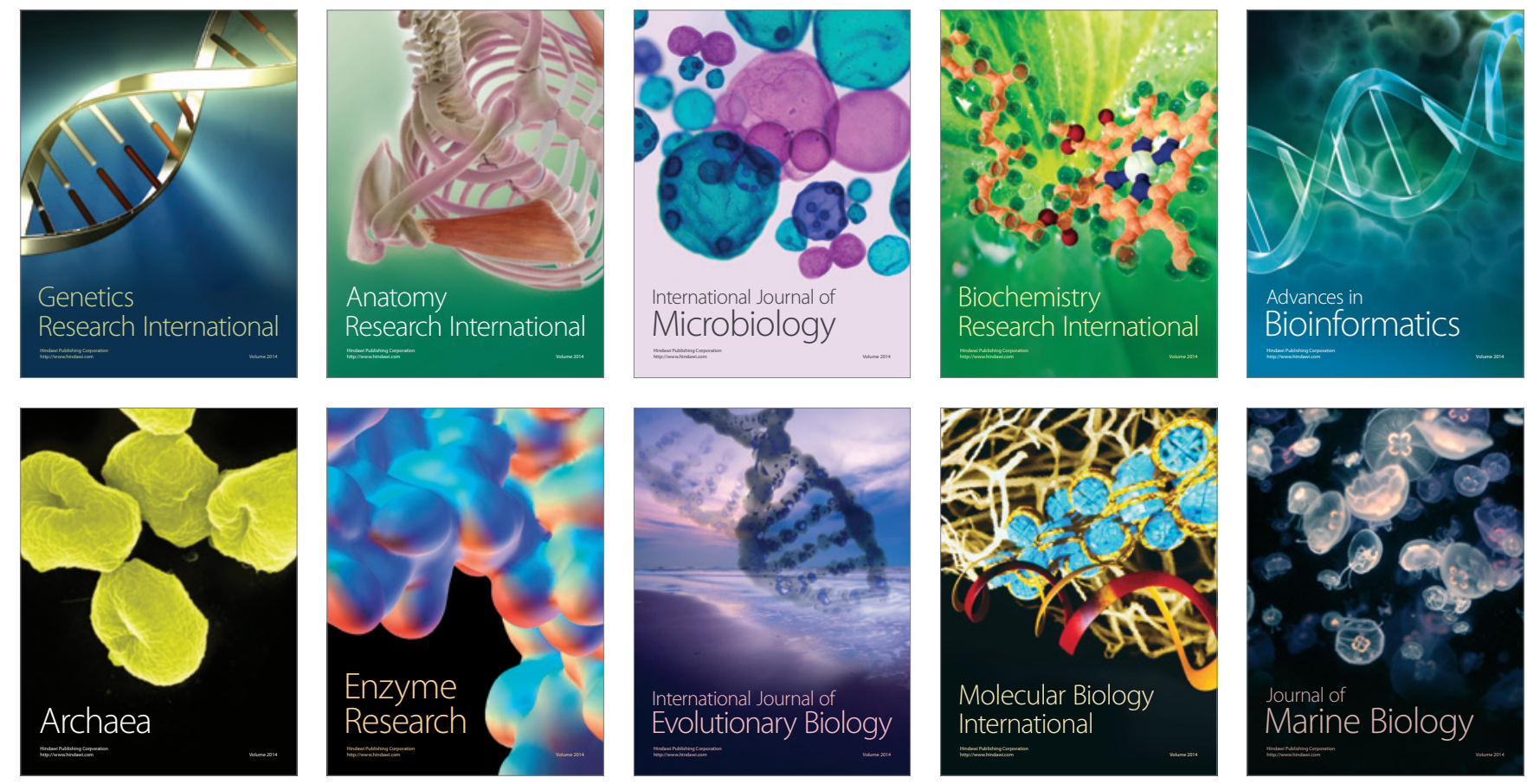